\title{
INVERSE CONTINUOUS WAVELET TRANSFORM IN WEIGHTED VARIABLE EXPONENT AMALGAM SPACES
}

\author{
Öznur KULAK ${ }^{1}$ and İsmail AYDIN ${ }^{2}$ \\ ${ }^{1}$ Amasya University, Faculty of Sciences and Letters, Department of Mathematics, Amasya, \\ TURKEY \\ ${ }^{2}$ Sinop University, Faculty of Sciences and Letters, Department of Mathematics, Sinop, \\ TURKEY
}

\begin{abstract}
The wavelet transform is an useful mathematical tool. It is a mapping of a time signal to the time-scale joint representation. The wavelet transform is generated from a wavelet function by dilation and translation. This wavelet function satisfies an admissible condition so that the original signal can be reconstructed by the inverse wavelet transform. In this study, we firstly give some basic properties of the weighted variable exponent amalgam spaces. Then we investigate the convergence of the $\theta$-means of $f$ in these spaces under some conditions. Finally, using these results the convergence of the inverse continuous wavelet transform is considered in these spaces.
\end{abstract}

\section{INTRODUCTION}

Recently, the variable exponent Lebesgue $L^{p(.)}\left(\mathbb{R}^{d}\right)$ spaces and a class of nonlinear problems with variable exponential growth have been new and interesting topics. The space has several applications, such as electrorheological fluids (see 31]), elastic mechanics (see [43]) and image processing model. Moreover, the spaces $L^{p(.)}\left(\mathbb{R}^{d}\right)$ and $L^{p}\left(\mathbb{R}^{d}\right)$ have many common properties, such as Banach space, reflexivity, separability, uniform convexity, Hölder inequalities and embeddings. One of the most important differences between these spaces is that the space $L^{p(\cdot)}\left(\mathbb{R}^{d}\right)$ is not translation invariant 27]. It is also well known that the maximal operator is bounded in $L^{p(.)}\left(\mathbb{R}^{d}\right)$. For more comprehensive information (see 10$],[12,[13$ and $[14]$ ).

The amalgam of $L^{p}$ and $l^{q}$ on the real line is the space $\left(L^{p}, l^{q}\right)$, which is also larger than the space $L^{p}$, consisting of functions which are locally in $L^{p}$ and have $l^{q}$

2020 Mathematics Subject Classification. 65T60, 42C40, 42B08, 43A15, 46B15, 46E30.

Keywords and phrases. Weighted variable exponent amalgam spaces, Inverse continuous wavelet transform, $\theta$-summability.

oznur.kulak@amasya.edu.tr-Corresponding author; iaydin@sinop.edu.tr

(D) 0000-0003-1433-3159; 0000-0001-8371-3158.

(C)2020 Ankara University Communications Faculty of Sciences University of Ankara-Series A1 Mathematics and Statistics 
behavior at infinity. Many different forms of amalgam spaces have been studied by some authors (see 25], 33], 24], 15 and [18]). Moreover, this space play important roles in recent developments in time frequency analysis and sampling theory, which are modern branches of harmonic analysis. Signal analysis and wireless communication issues are quite popular in amalgam spaces (see 20]).

Variable exponent amalgam spaces $\left(L^{p(.)}, l^{q}\right)$ and some basic properties, such as Banach function space, Hölder type inequalities, interpolation, bilinear multipliers and the boundedness of maximal operator, have been investigated recently. Some interesting articles have been published on this subject, but not many. So there are many open problems in this function spaces [5], 21], 26], 30], 22], 28], 3], 7], 2], 6.

The so called $\theta$-summation method is investigated by some authors, such as [36], [32], 38], 39], 40], 34, 8. The $\theta$-summation is defined by

$$
\sigma_{T}^{\theta} f(x)=\int_{\mathbb{R}^{d}} f(x-t) T^{n} \theta(T t) d t
$$

for an integrable function $\theta$ on $\mathbb{R}$. This summability is a generalized form of the wellknown summability methods, like Fejér, Riesz, Weierstrass, Abel, etc. by a suitable chosen of $\theta$. Feichtinger and Weisz ( [16, 17, 42]) showed that the $\theta$-means $\sigma_{T}^{\theta} f$ converges to $f$ almost everywhere and in norm as $T \rightarrow \infty$ for $f \in L^{p}\left(\mathbb{R}^{d}\right),\left(L^{p}, l^{q}\right)$. Also we characterize the points of the set of a.e. convergence as the Lebesgue points. Moreover, Uribe and Fiorenza [10], Szarvas and Weisz [34 obtained similar results for the space $L^{p(.)}\left(\mathbb{R}^{d}\right)$.

In this study we will discuss the convergence of the inverse continuous wavelet transform in weighted variable exponent amalgam spaces. Also, we investigate the convergence of the $\theta$-means of $f$ almost everywhere and in norm in these spaces under which conditions. Hence we obtain more general results with respect to 34].

\section{Weighted Variable Exponent Lebesgue and Amalgam spaces}

In this section we give some required definitions and information about wavelet transform and weighted variable exponent amalgam spaces.

Definition 1. Let $x \in \mathbb{R}^{d}, s \in \mathbb{R}$ and $s \neq 0$. The continuous wavelet transform is defined by

$$
W_{g} f(x, s)=|s|^{-\frac{d}{2}} \int_{\mathbb{R}^{d}} f(t) \overline{g\left(s^{-1}(t-x)\right)} d t=\left\langle f, T_{x} D_{s} g\right\rangle
$$

for $f$ and $g$, where $D_{s}$ is the dilation operator, and $T_{x}$ is the translation operator, i.e.,

$$
D_{s} f(t)=|s|^{-\frac{d}{2}} f\left(\frac{t}{s}\right) \text { and } T_{x} f(t)=f(t-x) \quad\left(x, t \in \mathbb{R}^{d}, 0 \neq s \in \mathbb{R}\right)
$$


[11], [19]. If $\eta$ is radial, non-increasing as a function on $(0, \infty)$, non-negative, bounded, $|f| \leq \eta$ and $\eta \in L^{1}\left(\mathbb{R}^{d}\right)$, then $\eta$ is a radial majorant of $f$. If in addition $\eta(.) \ln (||+2.) \in L^{1}\left(\mathbb{R}^{d}\right)$, then $\eta$ is a radial log-majorant of $f$.

Definition 2. A point $x \in \mathbb{R}^{d}$ is called a Lebesgue point $f \in L_{\text {loc }}^{1}\left(\mathbb{R}^{d}\right)$ if

$$
\lim _{h \rightarrow 0+}\left(\frac{1}{|B(0, h)|} \int_{B(0, h)}|f(x+u)-f(x)| d u\right)=0,
$$

where

$$
B(a, \delta)=\left\{x \in \mathbb{R}^{d}:\|x-a\|<\delta\right\} .
$$

Definition 3. Let $g^{*}(x)=\overline{g(-x)}$ be involution operator. Then the operators $\rho_{S} f$ and $\rho_{S, T} f$ are defined by

$$
\rho_{S} f=\int_{S}^{\infty} \int_{\mathbb{R}^{d}} W_{g} f(x, s) T_{x} D_{s} \gamma \frac{d x d s}{s^{d+1}}
$$

and

$$
\rho_{S, T} f=\int_{S}^{T} \int_{\mathbb{R}^{d}} W_{g} f(x, s) T_{x} D_{s} \gamma \frac{d x d s}{s^{d+1}},
$$

where $0<S<T<\infty$. Let define the operator $C_{g, \gamma}^{\prime}$ with

$$
C_{g, \gamma}^{\prime}=-\int_{\mathbb{R}^{d}}\left(g^{*} * \gamma\right)(x) \ln (|x|) d x .
$$

Then $C_{g, \gamma}^{\prime}$ is finite [29], where $g$ and $\gamma$ both have radial log-majorants.

Let $g$ and $\gamma$ be radial, i.e., $\int_{\mathbb{R}^{d}}\left(g^{*} * \gamma\right)(x) d x=0$. Assume that $g$ and $\gamma$ have a radial log-majorant. Then we get

$$
\lim _{S \rightarrow 0^{+}, T \rightarrow \infty} \rho_{S, T} f(x)=\lim _{S \rightarrow 0^{+}} \rho_{S} f(x)=C_{g, \gamma}^{\prime} f(x)
$$

at every Lebesgue point for any $f \in L^{p}\left(\mathbb{R}^{d}\right)(1 \leq p<\infty)$. The convergence is proved with respect to $L^{p}$-norm for $T=\infty, 29$. Under some similar conditions, Weisz has proved similar results 41].

Definition 4. Let $p($.$) be a measurable function from \mathbb{R}^{d}$ into $[1, \infty)$ (called a variable exponent on $\mathbb{R}^{d}$ ) satisfying the condition $1 \leq p^{-} \leq p(.) \leq p^{+}<\infty$, where

$$
p^{-}=\underset{x \in \mathbb{R}^{d}}{\operatorname{essinf}} p(x), \quad p^{+}=\underset{x \in \mathbb{R}^{d}}{\operatorname{ess} \sup p}(x) .
$$


The set $P\left(\mathbb{R}^{d}\right)$ denotes variable exponents on $\mathbb{R}^{d}$. Let $p(.) \in P\left(\mathbb{R}^{d}\right)$. The variable exponent Lebesgue spaces $L^{p(.)}\left(\mathbb{R}^{d}\right)$ consist of all measurable functions $f$ such that $\varrho_{p(.)}(\lambda f)<\infty$ for some $\lambda>0$, equipped with the Luxemburg norm

$$
\|f\|_{p(.)}=\inf \left\{\lambda>0: \varrho_{p(.)}\left(\frac{f}{\lambda}\right) \leq 1\right\},
$$

where

$$
\varrho_{p(.)}(f)=\int_{\mathbb{R}^{d}}|f(x)|^{p(x)} d x .
$$

If $p^{+}<\infty$, then $f \in L^{p(.)}\left(\mathbb{R}^{d}\right)$ iff $\varrho_{p(.)}(f)<\infty$. The space $\left(L^{p(.)}\left(\mathbb{R}^{d}\right),\|\cdot\|_{p(.)}\right)$ is a Banach space. If $p()=$.$p is a constant function, then the norm \|\cdot\|_{p(.)}$ coincides with the usual Lebesgue norm $\|\cdot\|_{p}$, [27. A measurable and locally integrable function $\omega: \mathbb{R}^{d} \longrightarrow(0, \infty)$ is called a weight function. The weighted modular is defined by

$$
\varrho_{p(.), \omega}(f)=\int_{\mathbb{R}^{d}}|f(x)|^{p(x)} \omega(x) d x .
$$

The space $L_{\omega}^{p(.)}\left(\mathbb{R}^{d}\right)$ is of all measurable functions such that $\|f\|_{L_{\omega}^{p(.)}\left(\mathbb{R}^{d}\right)}=\left\|f \omega^{\frac{1}{p(.)}}\right\|_{p(.)}<$ $\infty$. The dual space of $L_{\omega}^{p(.)}\left(\mathbb{R}^{d}\right)$ is $L_{\omega^{*}}^{q(.)}\left(\mathbb{R}^{d}\right)$, where $\frac{1}{p(.)}+\frac{1}{q(.)}=1$ and $\omega^{*}=$ $\omega^{1-q(.)}=\omega^{-\frac{1}{p(.)-1}}$.Also, $L_{\omega}^{p(.)}\left(\mathbb{R}^{d}\right)$ is a uniformly convex Banach space, thus reflexive for $1<p^{-} \leq p(.) \leq p^{+}<\infty$, [3], [4].

Definition 5. The maximal operator $M$ is defined by

$$
M(f)(x)=\sup _{r>0} \frac{1}{|B(x, r)|} \int_{B(x, r)}|f(y)| d y,
$$

for $f \in L_{\text {loc }}^{1}(\mathbb{R})$.

Hästö and Diening 23] defined the class $A_{p(.)}$ consists of those weights $\omega$ such that

$$
\|\omega\|_{A_{p(.)}}=\sup _{B \in \beta}|B|^{-p_{B}}\|\omega\|_{L^{1}(B)}\left\|\frac{1}{\omega}\right\|_{L^{\frac{p^{\prime}(.)}{p(.)}(B)}}<\infty
$$

where $\beta$ denotes the set of all balls in $\mathbb{R}^{d}, p_{B}=\left(\frac{1}{|B|} \int_{B} \frac{1}{p(x)} d x\right)^{-1}$ and $\frac{1}{p(.)}+\frac{1}{p^{\prime}(.)}=$ 1. If $p($.$) is a constant function, then A_{p(.)}=A_{p}$, where $A_{p}$ is ordinary Muckenhoupt class.

If $p($.$) satisfies the following inequality$

$$
|p(x)-p(y)| \leq \frac{C}{\log \left(e+\frac{1}{|x-y|}\right)}
$$


for all $x, y \in \mathbb{R}^{d}$, then $p($.$) provides the local log-Hölder continuity condition.$ Moreover, if the inequality

$$
\left|p(x)-p_{\infty}\right| \leq \frac{C}{\log (e+|x|)}
$$

holds for some $p_{\infty}>1, C>0$ and all $x \in \mathbb{R}^{d}$, then we say that $p($.$) satisfies$ the local log-Hölder decay condition. We denote by $P^{\log }\left(\mathbb{R}^{d}\right)$ the class of variable exponents which are log-Hölder continuous, i.e. which satisfy the local log-Hölder continuity condition and local log-Hölder decay condition [4], 37.

Let $p \in P^{\log }\left(\mathbb{R}^{d}\right)$ and $1<p^{-} \leq p(.) \leq p^{+}<\infty$. Then $M: L_{\omega}^{p(.)}\left(\mathbb{R}^{d}\right) \hookrightarrow$ $L_{\omega}^{p(.)}\left(\mathbb{R}^{d}\right)$ if and only if $\omega \in A_{p(.)}$ by Theorem 1.1 in 23 .

The space $L_{l o c, \omega}^{p(.)}\left(\mathbb{R}^{d}\right)$ is to be space of functions on $\mathbb{R}^{d}$ such that $f$ restricted to any compact subset $K$ of $\mathbb{R}^{d}$ belongs to $L_{w}^{p(.)}\left(\mathbb{R}^{d}\right)$.

In this study we take $d=1$, and define the weighted variable exponent amalgam spaces on $\mathbb{R}$.

Definition 6. Let $1 \leq p(),. q<\infty$ and $J_{k}=[k, k+1), k \in \mathbb{Z}$. The weighted variable exponent amalgam spaces $\left(L_{\omega}^{p(.)}, l^{q}\right)$ are defined by

$$
\left(L_{\omega}^{p(.)}, l^{q}\right)=\left\{f \in L_{l o c, \omega}^{p(.)}(\mathbb{R}):\|f\|_{\left(L_{\omega}^{p(.)}, l^{q}\right)}<\infty\right\}
$$

where $\|f\|_{\left(L_{\omega}^{p(.)}, l^{q}\right)}=\left(\sum_{k \in \mathbb{Z}}\left\|f \chi_{J_{k}}\right\|_{L_{\omega}^{p(.)}(\mathbb{R})}^{q}\right)^{\frac{1}{q}}$. If the weight $\omega$ is a constant function, then the space $\left(L_{\omega}^{p(.)}, l^{q}\right)$ coincides with $\left(L^{p(.)}, l^{q}\right)($ see [7], [26]).

In 2014, Meskhi and Zaighum showed that the maximal operator is bounded in weighted variable exponent amalgam spaces under some conditions [30].

Throughout this paper, we assume that $p(.) \in P^{\log }(\mathbb{R}), 1<p^{-} \leq p($. $p^{+}<\infty$ and $\omega \in A_{p(.)}$.

\section{3. $\theta$-Summability on the Weighted Variable Exponent Wiener AMALGAM SPACES}

Lemma 1. Let $1 \leq p(),. q<\infty$ and $0<c \leq \omega$. Then the inclusion $\left(L_{\omega}^{p(.)}, l^{q}\right) \subset$ $\left(L^{1}, l^{\infty}\right)$ holds.

Proof. Take any $f \in\left(L_{\omega}^{p(.)}, l^{q}\right)$. It is well known that $f \in\left(L_{\omega}^{p(.)}, l^{q}\right)$ if and only if $\left\{\|f\|_{L_{\omega}^{p(.)}[k, k+1)}\right\}_{k \in \mathbb{Z}} \in l^{q}$. If we use Proposition 3.5 in $|3|$ and the definition of $\|\cdot\|_{\left(L^{1}, l^{\infty}\right)}$, then we have $L_{\omega}^{p(\cdot)}[k, k+1) \hookrightarrow L_{\omega}^{1}[k, k+1) \hookrightarrow L^{1}[k, k+1), l^{q} \hookrightarrow l^{\infty}$ for $1 \leq p(),. q<\infty, 0<c \leq \omega$ and so

$$
\|f\|_{\left(L^{1}, l^{\infty}\right)}=\sup _{k \in \mathbb{Z}}\|f\|_{L^{1}[k, k+1)} \leq C \sup _{k \in \mathbb{Z}}\|f\|_{L_{\omega}^{p(\cdot)}[k, k+1)}
$$




$$
\leq C\left(\sum_{k \in \mathbb{Z}}\|f\|_{L_{\omega}^{p(.)}[k, k+1)}^{q}\right)^{\frac{1}{q}}=C\|f\|_{\left(L_{\omega}^{p(.)}, l^{q}\right)}<\infty .
$$

Hence we obtain that $f \in\left(L^{1}, l^{\infty}\right)$ and $\left(L_{\omega}^{p(.)}, l^{q}\right) \subset\left(L^{1}, l^{\infty}\right)$.

Theorem 1. Let $1 \leq q \leq p^{-} \leq p(.) \leq p^{+}<\infty$ and $0<c \leq \omega$. Then the inclusion

$$
\left(L_{\omega}^{p(\cdot)}, l^{q}\right) \hookrightarrow L_{\omega}^{q} \hookrightarrow L^{q},
$$

hold for all $f \in\left(L_{\omega}^{p(\cdot)}, l^{q}\right)$.

Proof. Let $f \in\left(L_{\omega}^{p(.)}, l^{q}\right)$ be given. Then we get $\left(L_{\omega}^{p(.)}, l^{q}\right) \hookrightarrow\left(L_{\omega}^{p^{-}}, l^{q}\right) \hookrightarrow$ $\left(L_{\omega}^{q}, l^{q}\right)=L_{\omega}^{q}$ by Proposition 3.5 in 3 and 24 . Hence we have that there exists a $C>0$ such that the inequality

$$
\|f\|_{L^{q}} \leq C\|f\|_{\left(L_{\omega}^{p(.)}, l^{q}\right)}
$$

holds for any $f \in\left(L_{\omega}^{p(.)}, l^{q}\right)$. This completes the proof.

Definition 7. Let $\theta \in L^{1}(\mathbb{R})$ be radial function. The $\theta$-means of $f \in\left(L_{\omega}^{p(.)}, l^{q}\right)$ is defined by

$$
\sigma_{T}^{\theta} f(x):=\left(f * \theta_{T}\right)(x)=\int_{\mathbb{R}} f(x-t) \theta_{T}(t) d t,
$$

where

$$
\theta_{T}(t):=T^{d} \theta(T t),(x \in \mathbb{R}, T>0) .
$$

Theorem 2. Let $1 \leq p(),. q<\infty$ and $0<c \leq \omega$. Assume that $\theta$ has radial majorant. Then;

i) The limit

$$
\lim _{T \rightarrow \infty} \sigma_{T}^{\theta} f(x)=\int_{\mathbb{R}} \theta(y) d y \cdot f(x)
$$

is valid for any Lebesgue point of $f \in\left(L_{\omega}^{p(.)}, l^{q}\right)$.

ii) If in addition $1 \leq q \leq p^{-} \leq p(.) \leq p^{+}<\infty$, then the following limit equality

$$
\lim _{T \rightarrow 0^{+}} \sigma_{T}^{\theta} f(x)=0
$$

is available for all $f \in\left(L_{\omega}^{p(\cdot)}, l^{q}\right)$ and $x \in \mathbb{R}$. 
Proof. i) Let $x \in \mathbb{R}$ be a Lebesgue point of $f$. Since there exists the inclusion $\left(L_{\omega}^{p(.)}, l^{q}\right) \subset\left(L^{1}, l^{\infty}\right)$ by Lemma 1 , we write that

$$
\lim _{T \rightarrow \infty} \sigma_{T}^{\theta} f(x)=\int_{\mathbb{R}^{n}} \theta(y) d y . f(x)
$$

for $f \in\left(L_{\omega}^{p(.)}, l^{q}\right)$ by Theorem 2.2 in 34 .

ii) Take any $f \in\left(L_{\omega}^{p(.)}, l^{q}\right)$ and $x \in \mathbb{R}$. By Theorem 1 and Theorem 2.3 in 34 , we have that $f \in L^{q}(\mathbb{R})$ and

$$
\lim _{T \rightarrow 0^{+}} \sigma_{T}^{\theta} f(x)=0
$$

Proposition 1. $C_{c}(\mathbb{R})$, which consists of continuous functions on $\mathbb{R}$ whose support is compact, is dense in $\left(L_{\omega}^{p(.)}, l^{q}\right)$ for $1 \leq p(),. q<\infty$ (see Proposition 2.9 in [6]).

Theorem 3. For all $f \in\left(L_{\omega}^{p(.)}, l^{q}\right)$ the following statements are valid:

i) $\left\|\sigma_{T}^{\theta} f\right\|_{\left(L_{\omega}^{p(.)}, l^{q}\right)} \leq C\|f\|_{\left(L_{\omega}^{p(.)}, l^{q}\right)}(T>0)$.

ii) $\lim _{T \rightarrow \infty} \sigma_{T}^{\theta} f=\int_{\mathbb{R}} \theta(x) d x . f$ in the $\left(L_{\omega}^{p(.)}, l^{q}\right)$-norm.

iii) $\lim _{T \rightarrow 0^{+}} \sigma_{T}^{\theta} f=0$ in the $\left(L_{\omega}^{p(.)}, l^{q}\right)$-norm.

Proof. i) It is well known that the maximal operator is bounded in $\left(L_{\omega}^{p(.)}, l^{q}\right) 30$. Then we have that

$$
\left\|\sigma_{T}^{\theta} f\right\|_{\left(L_{\omega}^{p(\cdot), l^{q}}\right)} \leq C\|f\|_{\left(L_{\omega}^{p(\cdot)}, l^{q}\right)}(T>0)
$$

for all $f \in\left(L_{\omega}^{p(.)}, l^{q}\right)$ by Theorem 2.1 in 34 .

ii) Also, if we follow Theorem 3.8 in 34, Theorem 2.3 in 9, Theorem 5.11 in [10, and Theorem 8 in [1, then we have that

$$
\lim _{T \rightarrow \infty} \sigma_{T}^{\theta} f=\int_{\mathbb{R}} \theta(x) d x . f
$$

in the $\left(L_{\omega}^{p(.)}, l^{q}\right)$-norm.

iii) Let $\epsilon>0$ be given. Using Proposition 1, it is obtained that the following inequality

$$
\|f-g\|_{\left(L_{\omega}^{p(\cdot)}, l^{q}\right)}<\epsilon
$$


is valid for $g \in C_{c}(\mathbb{R})$, whose compact support supp $g$ is $K$. Using i) and Proposition 2 in 7 , we have that

$$
\begin{aligned}
\left\|\sigma_{T}^{\theta} f\right\|_{\left(L_{\omega}^{p(.)}, l^{q}\right)} & \leq\left\|\sigma_{T}^{\theta}(f-g)\right\|_{\left(L_{\omega}^{p(\cdot)}, l^{q}\right)}+\left\|\sigma_{T}^{\theta} g\right\|_{\left(L_{\omega}^{p(.)}, l^{q}\right)} \\
& <C \epsilon+|S(K)|^{\frac{1}{q}}\left\|\sigma_{T}^{\theta} g\right\|_{L_{\omega}^{p(.)}(K)} .
\end{aligned}
$$

Also using Theorem 3.8 in 34 , we get the limit

$$
\lim _{T \rightarrow 0^{+}}\left\|\sigma_{T}^{\theta} g\right\|_{L_{\omega}^{p(.)}(K)}=0 .
$$

So this completes the proof.

\section{Convergence of $\rho_{S}$ And $\rho_{S, T}$}

Theorem 4. Assume that $g, \gamma$ have radial log-majorants and $\int_{\mathbb{R}}\left(g^{*} * \gamma\right)(x) d x=0$. If $\omega \in A_{1}$ and $0<c \leq \omega$, then for all $f \in\left(L_{\omega}^{p(.)}, l^{q}\right)$ the following relation holds;

$$
\rho_{S} f=\sigma_{\frac{1}{S}}^{\theta} f,(S>0)
$$

where

$$
\theta(y)=\int_{1}^{\infty}\left(g^{*} * \gamma\right)\left(\frac{y}{u}\right) \frac{1}{u^{n+1}} \chi_{B(0,1)}(y) d u-\int_{0}^{1}\left(g^{*} * \gamma\right)\left(\frac{y}{u}\right) \frac{1}{u^{n+1}} \chi_{\mathbb{R}^{n} \backslash B(0,1)}(y) d u .
$$

Proof. Let $f \in L_{\omega}^{1} \cap\left(L_{\omega}^{p(.)}, l^{q}\right)$ and $y \in \mathbb{R}$. Then we have decomposition of $\rho_{S} f(y)$ as

$$
\begin{aligned}
\rho_{S} f(y)= & \int_{S} \int_{\mathbb{R}} \frac{1}{s^{3}} \int_{\mathbb{R}} f(t) \overline{\left(\frac{t-x}{s}\right)} \gamma\left(\frac{y-x}{s}\right) d t d x d s \\
= & \int_{S}^{\infty} \int_{|y-t|<S} \frac{1}{s^{3}} \int_{\mathbb{R}} f(t) \overline{g\left(\frac{t-x}{s}\right)} \gamma\left(\frac{y-x}{s}\right) d x d t d s \\
& -\int_{0}^{S} \int_{|y-t| \geq S} \frac{1}{s^{3}} \int_{\mathbb{R}} f(t) \overline{g\left(\frac{t-x}{s}\right)} \gamma\left(\frac{y-x}{s}\right) d x d t d s \\
& +\int_{0}^{\infty} \int_{|y-t| \geq S} \frac{1}{s^{3}} \int_{\mathbb{R}} f(t) \overline{g\left(\frac{t-x}{s}\right)} \gamma\left(\frac{y-x}{s}\right) d x d t d s \\
= & I-I I+I I I
\end{aligned}
$$


by from [29], 34. Also it is well known that

$$
I=\left(f * \varphi_{\frac{1}{S}}\right)(y) \text { and } I I=\left(f * \psi_{\frac{1}{S}}\right)(y),
$$

where

$$
\varphi(t)=\int_{1}^{\infty}\left(g^{*} * \gamma\right)\left(\frac{t}{u}\right) \frac{1}{u^{n+1}} \chi_{B(0,1)}(t) d u
$$

and

$$
\psi(t)=\int_{0}^{1}\left(g^{*} * \gamma\right)\left(\frac{t}{u}\right) \frac{1}{u^{n+1}} \chi_{\mathbb{R}^{n} \backslash B(0,1)}(t) d u
$$

by proof of Theorem 1.1 in $[29$. On the other hand, Szarvas and Weisz proved that $\varphi$ and $\psi$ have radial majorants by Theorem 5.1 in 34 in case $g$ and $\gamma$ have radial $\log$-majorants. Since $g, \gamma$ have radial log-majorants, $f \in L_{\omega}^{1}, \omega \in A_{1}$ and

$$
\int_{\mathbb{R}}\left(g^{*} * \gamma\right)(x) d x=0,
$$

then we have

$$
\begin{aligned}
I I I & =\int_{0}^{\infty} \int_{|y-t| \geq S} \frac{1}{s^{3}} \int_{\mathbb{R}} f(t) \overline{\left(\frac{t-x}{s}\right)} \gamma\left(\frac{y-x}{s}\right) d x d t d s \\
& =\frac{1}{\omega_{0}} \int_{|y-t| \geq S} \frac{f(t)}{|y-t|} \int_{\mathbb{R}}\left(g^{*} * \gamma\right)(u) d u d t=0
\end{aligned}
$$

by Lemma 2.5 in 29 . Therefore we get

$$
\begin{aligned}
\rho_{S} f(y) & =\left(f * \varphi_{\frac{1}{S}}\right)(y)-\left(f * \psi_{\frac{1}{S}}\right)(y)+0 \\
& =f *\left(\varphi_{\frac{1}{S}}-\psi_{\frac{1}{S}}\right)(y)=f * \theta_{\frac{1}{S}}(y)=\sigma_{\frac{1}{S}}^{\theta} f(y),
\end{aligned}
$$

where

$$
\begin{aligned}
\theta(y) & =\varphi(y)-\psi(y) \\
& =\int_{1}^{\infty}\left(g^{*} * \gamma\right)\left(\frac{y}{u}\right) \frac{1}{u^{n+1}} \chi_{B(0,1)}(y) d u-\int_{0}^{1}\left(g^{*} * \gamma\right)(y) \frac{1}{u^{n+1}} \chi_{\mathbb{R}^{n} \backslash B(0,1)}(y) d u .
\end{aligned}
$$

If $\varphi, \psi$ have radial majorants, then $\theta=\varphi-\psi$ have radial majorant, that is, $\theta$ is a non-negative and non-increasing function, and belongs to the space $L^{1} \cap L^{\infty}$. So it is obtained that

$$
\|\theta\|_{\left(L^{\infty}, l^{1}\right)}=\sum_{k \in \mathbb{Z}}\left\|\theta \chi_{[k, k+1)}\right\|_{\infty} \leq \sum_{k \in \mathbb{Z}} \theta(k)<\infty
$$

and $\theta \in\left(L^{\infty}, l^{1}\right)$. Then using Hölder inequality and Lemma 1, we have 


$$
\begin{aligned}
\left|\rho_{S} f(y)\right| & =\left|\sigma_{\frac{1}{S}}^{\theta} f(y)\right| \leq \frac{1}{S} \int_{\mathbb{R}}|f(y-t)|\left|\theta\left(\frac{t}{S}\right)\right| d t \\
& \leq C\|f\|_{\left(L^{1}, l^{\infty}\right)}\|\theta\|_{\left(L^{\infty}, l^{1}\right)} \\
& \leq C\|f\|_{\left(L_{\omega}^{p(\cdot)}, l^{q}\right)}\|\theta\|_{\left(L^{\infty}, l^{1}\right)} .
\end{aligned}
$$

Hence the function $\rho_{S}$ is linear and bounded from $L_{\omega}^{1} \cap\left(L_{\omega}^{p(.)}, l^{q}\right)$ to $\mathbb{C}$. Also, it is well known that the inclusion $C_{c} \subset L_{\omega}^{1} \cap\left(L_{\omega}^{p(.)}, l^{q}\right) \subset\left(L_{\omega}^{p(.)}, l^{q}\right)$. Since $C_{c}$ is dense in $\left(L_{\omega}^{p(.)}, l^{q}\right)$ 6, then we find that $L_{\omega}^{1} \cap\left(L_{\omega}^{p(.)}, l^{q}\right)$ is dense in $\left(L_{\omega}^{p(.)}, l^{q}\right)$. Therefore, from the density principle, the function $\rho_{s}$ is extended from $\left(L_{\omega}^{p(.)}, l^{q}\right)$ to $\mathbb{C}$. This completes the proof.

Theorem 5. Let $f \in\left(L_{\omega}^{p(.)}, l^{q}\right)$. Moreover, assume that $g, \gamma$ have radial logmajorants and $\int_{\mathbb{R}}\left(g^{*} * \gamma\right)(x) d x=0$. If $\omega \in A_{1}$ and $0<c \leq \omega$, then

i) $\lim _{S \rightarrow 0^{+}} \rho_{S} f(x)=C_{g, \gamma}^{\prime} f(x)$

for any Lebesgue point of the function $f$.

ii) If in addition $1 \leq q \leq p(.) \leq p^{+}<\infty$, then

$\lim _{S \rightarrow 0^{+}, T \rightarrow \infty} \rho_{S, T} f(x)=C_{g, \gamma}^{\prime} f(x)$

for any Lebesgue point of the function $f$.

Proof. i) Since $p(.) \in P^{\log }(\mathbb{R})$ and $1<p^{-} \leq p(.) \leq p^{+}<\infty$, then $A_{1} \subset A_{p(.)}$ 4. By Theorem 2 and Theorem 4, we deduce that

$$
\lim _{S \rightarrow 0^{+}} \rho_{S} f(x)=\lim _{S \rightarrow 0^{+}} \sigma_{\frac{1}{S}}^{\theta} f(x)=\int_{\mathbb{R}} \theta(y) d y f(x)
$$

for all Lebesgue points of $f \in\left(L_{\omega}^{p(.)}, l^{q}\right)$. On the other hand, using Theorem 5.2 in 34 , we have that $\int_{\mathbb{R}} \theta(y) d y=C_{g, \gamma}^{\prime}$ and

$$
\lim _{S \rightarrow 0^{+}} \rho_{S} f(x)=C_{g, \gamma}^{\prime} f(x) .
$$

ii) By Theorem 5.2 in 34 we can write the equality $\rho_{S, T} f(x)=\rho_{S} f(x)-\rho_{T} f(x)$ for $x \in \mathbb{R}$. Then using (i), Theorem 2 and Theorem 4 , we obtain that

$$
\begin{aligned}
\lim _{S \rightarrow 0^{+}, T \rightarrow \infty} \rho_{S, T} f(x) & =\lim _{S \rightarrow 0^{+}} \rho_{S} f(x)-\lim _{T \rightarrow \infty} \rho_{T} f(x) \\
& =\lim _{S \rightarrow 0^{+}} \sigma_{\frac{1}{S}}^{\theta} f(x)-\lim _{T \rightarrow \infty} \sigma_{\frac{1}{T}}^{\theta} f(x) \\
& =C_{g, \gamma}^{\prime} f(x)-0=C_{g, \gamma}^{\prime} f(x) .
\end{aligned}
$$


Corollary 1. Assume that $g, \gamma$ have radial log-majorants, $\int_{\mathbb{R}}\left(g^{*} * \gamma\right)(x) d x=0$. If $\omega \in A_{1}$ and $0<c \leq \omega$, then the following statements are valid for any $f \in$ $\left(L_{\omega}^{p(.)}, l^{q}\right)$

i) $\lim _{S \rightarrow 0^{+}} \rho_{s} f(x)=C_{g, \gamma}^{\prime} f(x)$ a.e.

ii) If in addition $1 \leq q \leq p(.) \leq p^{+}<\infty$, then

$\lim _{S \rightarrow 0^{+}, T \rightarrow \infty} \rho_{s, T} f(x)=C_{g, \gamma}^{\prime} f(x)$ a.e.

Proof. Let $f \in\left(L_{\omega}^{p(.)}, l^{q}\right)$. Then by Lemma 1 , we have $f \in\left(L^{1}, l^{\infty}\right)$. It is known that if $f \in\left(L^{1}, l^{\infty}\right)$, then real numbers almost everywhere is a Lebesgue point of $f$, 16], 17. Hence by the Theorem 5, we complete the proof.

Theorem 6. Assume that $g, \gamma$ have radial log-majorants and $\int_{\mathbb{R}}\left(g^{*} * \gamma\right)(x) d x=0$. If $\omega \in A_{1}$ and $0<c \leq \omega$, then the following results

i) $\lim _{S \rightarrow 0^{+}} \rho_{s} f=C_{g, \gamma}^{\prime} f$,

ii) $\underset{S \rightarrow 0^{+}, T \rightarrow \infty}{ } \rho_{s, T} f=C_{g, \gamma}^{\prime} f$

are satisfied in the $\left(L_{\omega}^{p(.)}, l^{q}\right)$-norm for all $f \in\left(L_{\omega}^{p(.)}, l^{q}\right)$.

Proof. i) Using $\omega \in A_{1} \subset A_{p(.)}$, Theorem 3 and Theorem 4, we have

$$
\lim _{S \rightarrow 0^{+}} \rho_{S} f=\lim _{S \rightarrow 0^{+}} \sigma_{\frac{1}{S}}^{\theta}=\int_{\mathbb{R}} \theta(y) d y f
$$

in the $\left(L_{\omega}^{p(.)}, l^{q}\right)$-norm for all $f \in\left(L_{\omega}^{p(.)}, l^{q}\right)$. On the other hand, since $\int_{\mathbb{R}} \theta(y) d y=$ $C_{g, \gamma}^{\prime}$, then we obtain that

$$
\lim _{S \rightarrow 0^{+}} \rho_{S} f=C_{g, \gamma}^{\prime} f
$$

in the $\left(L_{\omega}^{p(.)}, l^{q}\right)$-norm.

ii) Since $\rho_{S, T} f=\rho_{S} f-\rho_{T} f$, then we have that

$$
\begin{aligned}
\lim _{S \rightarrow 0^{+}, T \rightarrow \infty} \rho_{s, T} f & =\lim _{S \rightarrow 0^{+}} \rho_{S} f-\lim _{T \rightarrow \infty} \rho_{T} f \\
& =\lim _{S \rightarrow 0^{+}} \sigma_{\frac{1}{S}}^{\theta} f-\lim _{T \rightarrow \infty} \sigma_{\frac{1}{T}}^{\theta} f=C_{g, \gamma}^{\prime} f
\end{aligned}
$$

in the $\left(L_{\omega}^{p(.)}, l^{q}\right)$-norm by (i), Theorem 3 and Theorem 4 .

\section{REFERENCES}

[1] Aydın, I., Unal, C., The Kolmogorov-Riesz theorem and some compactness criterions of bounded subsets in weighted variable exponent amalgam and Sobolev spaces, Collectanea Mathematica, (2019), https://doi.org/10.1007/s13348-019-00262-5, 1-19.

[2] Aydın, I., Unal, C., On some multipliers of vector-valued amalgam spaces, Int. Journal of Pure and Appl. Math., 116 (2) (2017), 547-557. 
[3] Aydın, I., On variable exponent amalgam spaces, Analele Stiint. Univ., 20(3) (2012), 5-20.

[4] Aydın, I., Weighted variable Sobolev spaces and capacity, J. Funct. Space Appl., Volume 2012, Article ID 132690, 17 pages, doi:10.1155/2012/132690.

[5] Aydın, I., Gürkanll, A.T., Weighted variable exponent amalgam spaces $W\left(L^{p(x)} ; L_{w}^{q}\right)$, Glas. Mat., 47(67) (2012), 165-174.

[6] Aydın, I., Unal, C., Birkhoff's ergodic theorem for weighted variable exponent amalgam spaces, Applications and Applied Mathematics: An International Journal (AAM), Special Issue No. 3 (2019), 1-10.

[7] Aydin, I., On vector-valued classical and variable exponent amalgam spaces, Commun. Fac. Sci. Univ. Ank. Series A1, 66 (2) (2017), 100-114.

[8] Butzer, P.L., Nessel, R.J., Fourier Analysis and Approximation, Academic Press, NewyorkLondon, Volume 1, 1971.

[9] Cruz-Uribe, D., Fiorenza, A., Approximate identities in variable Lp spaces, Math. Nach., 280 (2007), 256-270.

[10] Cruz-Uribe, D., Fiorenza, A., Variable Lebesgue Spaces, Foundations and harmonic analysis, New York, NY, Birkhauser/Springer, 2013.

[11] Daubechies, I., Ten Lectures on Wavelets, SIAM, Philadelphia, 1992.

[12] Diening, L., Maximal function on generalized Lebesgue spaces $L^{p(.)}$, Mathematical Inequalities and Applications, 7 (2004), 245-253.

[13] Diening, L., Harjulehto, P., Hästö, P., Ružicka, M., Lebesgue and Sobolev Spaces with Variable Exponents, Lecture Notes in Math. 2017, Springer, Berlin, 2011.

[14] Fan, X., Zhao, D., On the spaces $L^{p(x)}(\Omega)$ and $W^{k, p(x)}(\Omega)$, J. Math. Anal. Appl., 263 (2) (2001), 424-446.

[15] Feichtinger, H. G., Banach convolution algebras of Wiener type. In: Functions, Series, Operators, Proc. Conf. Budapest 38, Colloq. Math. Soc., Janos Bolyai, (1980),509-524.

[16] Feichtinger, H. G., Weisz, F., The Segal algebra $S_{0}\left(\mathbb{R}^{d}\right)$ and norm summability of Fourier series and Fourier Transforms, Monatshefte Mth., 148 (2006), 333-349.

[17] Feichtinger, H. G., Weisz, F., Wiener amalgams and pointwise summability of Fourier Transforms and Fourier series, Math. Proc. Camb. Phil. Soc., 140 (2006), 509-536.

[18] Fournier, J.J., Stewart, J., Amalgams of $L^{p}$ and $l^{q}$, Bull. Amer. Math. Soc., 13 (1985), 1-21.

[19] Gröchenig, K., Foundations of Time-Frequency Analysis, Birkhauser, Boston, 2001.

[20] Gröchenig, K., Heil, C., Okoudjou, K., Gabor analysis in weighted amalgam spaces, Sampl. Theory Signal Image Process., Int. J., 1 (2002), 225-259.

[21] Gürkanlı, A.T., Aydın, I., On the weighted variable exponent amalgam space $W\left(L^{p(x)} ; L_{m}^{q}\right)$, Acta Math. Sci., 4(34B) (2014), 1-13.

[22] Gürkanlı, A.T., The amalgam spaces $W\left(L^{p(x)} ; L^{\left\{p_{n}\right\}}\right)$ and boundedness of Hardy-Littlewood maximal operators, Current Trends in Analysis and Its Applications, Proceedings of the 9th ISAAC Congress, Krakow (2013).

[23] Hästö, P., Diening, L., Muckenhoupt weights in variable exponent spaces, preprint, http://www.helsinki.fi/ pharjule/varsob/publications.shtml.

[24] Heil, C., An introduction to weighted Wiener amalgams. In: Wavelets and Their Applications, Allied Publishers, New Delhi, (2003), 183-216.

[25] Holland, F., Harmonic analysis on amalgams of $L^{p}$ and $l^{q}$, J. London Math. Soc., 2(10) (1975), 295-305.

[26] Kokilashvili, V., Meskhi, A., Zaighum, M.A., Weighted kernel operators in variable exponent amalgam spaces, J. Inequal. Appl., (2013), DOI:10.1186/1029-242X-2013-173.

[27] Kovacik, O., Rakosnik, J., On spaces $L^{p(x)}$ and $W^{k, p(x)}$, Czech. Math. J., 41(116) (1991), $592-618$.

[28] Kulak, O., Gürkanl,, A.T., Bilinear multipliers of weighted Wiener amalgam spaces and variable exponent Wiener amalgam spaces, J. Inequal. Appl., (2014), 476. 
[29] Li, K., Sun, W., Pointwise convergence of the Calderon reproducing formula, J. Fourier Anal. Appl., 18 (2012), 439-455.

[30] Meskhi, A., Zaighum, M.A., On the boundedness of maximal and potential operators in variable exponent amalgam spaces, Journal of Mathematical Inequalities, 8(1) (2014), 123152.

[31] Ruzicka, M., Electrorheological fluids: modeling and mathematical theory, Springer-Verlag, Berlin, 2000.

[32] Simon, P., $(C, \alpha)$ summability of Walsh-Kaczmarz-Fourier Series, J. Approx. Theory, 127(1) (2004), 39-60.

[33] Squire, M.L.T., Amalgams of $L^{p}$ and $l^{q}, \mathrm{PhD}$, McMaster University, 1984.

[34] Szarvas, K., Weisz, F., Continuous wavelet transform in variable Lebesgue spaces, Stud. Univ. Babes-Bolyai Math., 59(4) (2014), 497-512.

[35] Szarvas, K., Variable Lebesgue spaces and continuous wavelet transforms, Acta Mathematica Academiae Paedagogicae Nyiregyhaziensis, 32 (2016), 313-325.

[36] Trigub, R.M., Bellinsky, E.S., Fourier Analysis and Approximation of Function, Kluwer Academic Publishers, Dordrecht, 2004.

[37] Unal, C., Aydın, I., On some properties of the space $L_{w}^{p}\left(\mathbb{R}^{n}\right) \cap L_{\vartheta}^{q(.)}\left(\mathbb{R}^{n}\right)$, Cankaya University Journal of Science and Engineering, 13(2) (2016), 001-010.

[38] Weisz, F., Summability of multi-dimensional Fourier series and Hardy spaces, Mathematics and its Applications, Kluwer Academic Publishers, Dordrecht, 541, 2002.

[39] Weisz, F., Summability of multi-dimensional trigonometric Fourier series. Surv Approx Theory, 7 (2012), 1-179.

[40] Weisz, F., Convergence of the inverse continuous wavelet transform in Wiener amalgam spaces, Journal of Analysis, 35 (2015), 33-46.

[41] Weisz, F., Inversion formulas for the continuous wavelet transform, Acta. Math. Hungar., 138 (2013), 237-258.

[42] Weisz, F., Pointwise convergence in Pringsheim's sense of the summability of fourier transforms on Wiener amalgam spaces, Monatsh Math., 175(1) (2014), 143-160.

[43] Zhikov, V.V., Averaging of functionals of the calculus of variations and elasticity theory, Izv. Akad. Nauk SSSR Ser. Mat., 29(4) (1987), 33-66. 